\title{
Numerical Simulation of P3HT Based Organic Light Emitting Diode
}

\author{
Shilpi Raj and A.D.D. Dwivedi
}

\section{Department of Electrical and Electronics Engineering, Poornima University Jaipur, India}

\begin{abstract}
Recently, organic light emitting diodes (OLEDs) have become quite popular in the field of microelectronics like optoelectronics application, modulated light source and internet connection etc. Owing to the advantages of solid-state, self-emission, full color capability and flexibility, OLED has been recognized as one of the most promising flat panel display technology. This paper presents the results of single polymer layer OLED (ITO/P3HT/Ca) 1D and 2D-numerical simulation and analysis using Silvaco TCAD tools, which is a physically-based simulator. The physically-based simulation of the electrical and optical characteristics, such as luminescence power, current density versus electric field, current versus voltage and I-V characteristics for different thicknesses and doping concentration of emitting layer is presented. The distribution of Langevin recombination inside the structure is also presented and discussed for better understanding of physical processes and operation principal of OLED.
\end{abstract}

Keywords: Organic light emitting diode, Atlas, Silvaco TCAD, Simulation, P3HT.

\section{INTRODUCTION}

In last decade enormous research has been done by various researchers all over the world in the field of organic devices, OLEDs are one of them. In particular, organic light emitting diodes (OLEDs) gradually over rule the inorganic diodes due to the following advantages. OLEDs are brighter, flexible, cheaper, lightweight, transparent, wide view angle and provide faster response than inorganic ones [1]. OLEDs has huge range of application which includes fabrication of thin and flexible displays for TV, GPS navigators, digital cameras, portable media players, car radios and nightvision devices among others. OLEDs have been widely used long ago in most Motorola and Samsung color mobile phones, as well as some LG, Nokia and Sony Ericsson models [1]. Moreover, OLEDs are quite attractive as optoelectronic applications for communication purposes. They are widely used as modulated light sources (fiber optic signal transmission, remote control devices, light phones).

In this paper, single layer polymer OLED is investigated and its electrical optical characteristics obtained through the Technology Computer Aided Design (TCAD) software ATLAS from Silvaco [2]. ATLAS is a physics-based, 2D and 3D device simulator that predicts the electrical behavior of specified semiconductor structures and provides insight into the internal physical mechanisms associated with device operation. It gives detailed knowledge about the components included in device during operation like

"Address correspondence to this author at the Department of Electrical and Electronics Engineering, Poornima University Jaipur, India;

E-mail: shilpisweet.raj@gmail.com, adddwivedi@gmail.com two-dimensional profile of carrier concentration, electric field and current density profiles etc. Simulation and modeling of OLEDs within the framework of TCAD can provide the necessary information about device characteristics, also it helps to look inside the structure and allow us to avoid the long and costly device optimization process by numerous experimental runs. Technology and device modeling with a help of Technology CAD are widely used in organic electronic components development and manufacturing and many scientists around the world are interested in the investigations in this sphere [3-30].

The rest of the paper is organized as following. Section II contain general information about OLEDs, their structure, materials, and operating principles, describes the process of OLED simulation including the building of device structure, choosing the physical models and getting the distribution of Langevin recombination. Section III presents the simulation results and electrical and optical characteristics. Section IV draws the conclusions from this work.

\section{SIMULATION}

\section{A. Device Structure}

The simplest OLED structure consists only one active polymer layer/emissive layer (EML) sandwiched between two charged electrodes, anode and cathode. The active layer is composed by conducting polymer, e.g. polythiophenes (Pts), poly p-phenylene vinylene (PPV), poly $\mathrm{N}$ vinylcarbazole (PVK), or polyaniline (PANI).

The top electrode of OLED (cathode) is a metallic mirror with high reflectivity made of calcium and bottom 
electrode (anode) is indium tin oxide (ITO), which is transparent. The active layer is made up of $\mathrm{P} 3 \mathrm{HT}$. The electrical circuit diagram and structural diagram of OLED is shown in Figure 1 and 2 respectively.

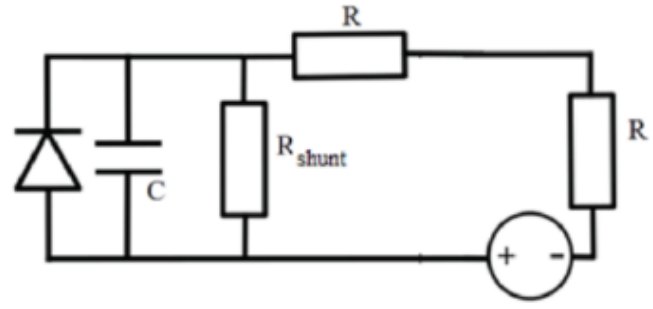

Figure 1: Electrical circuit diagram of OLED.

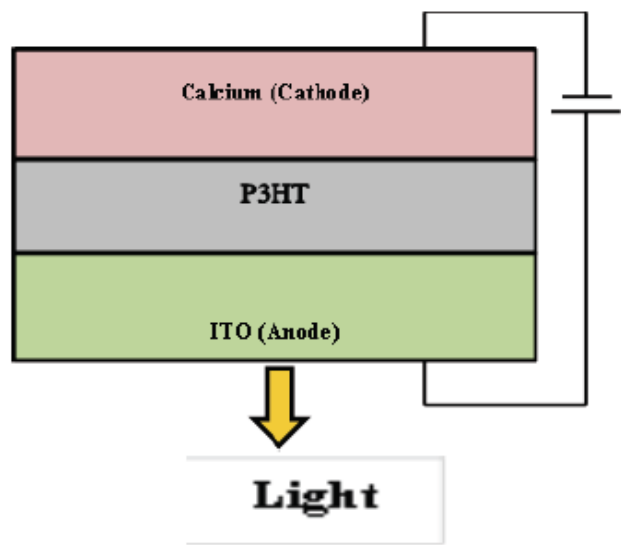

Figure 2: Structural diagram of OLED.

Poly(3-hexylthiophene) P3HT is a regioregular (Describing a polymer in which each repeat unit is derived from the same isomer of the monomer) semiconducting polymer. It is used in organic electronics primarily because of its regular end-to-end arrangement of side chain, which allows efficient $\boldsymbol{\Pi}-\boldsymbol{\Pi}$ stacking of the conjugated backbones.

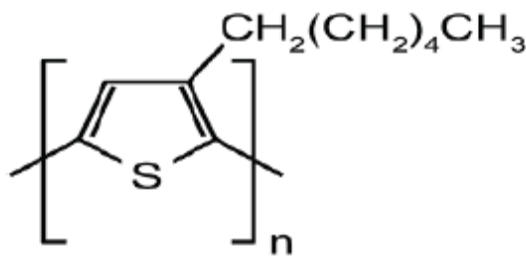

Figure 3: Molecular structure of $\mathrm{P} 3 \mathrm{HT}$.

\section{B. Operating Principle}

When a voltage is applied to the electrodes the charges start moving in the device under the influence of the electric field. Electrons are injected by the cathode into the lowest unoccupied molecular orbital
(LUMO) of the adjacent molecules and holes are injected by the anode into the highest occupied molecular orbital (HOMO). Electrons leave the cathode and holes migrate from the anode in opposite direction. A fraction of them recombines and thus leads to excitons formation, some of which decay radiatively to the ground state by spontaneous light emission. Therefore, the electrical power applied between the anode and cathode is transformed into light.

For modeling and simulation of OLED structure, a commercially available device simulator ATLAS was used which provides general capabilities for accurate simulation of organic devices. Here we firstly define a mesh of structure (a series of vertical and horizontal lines and the spacing between them) using Atlas syntax. A good mesh is important for accuracy and numerical efficiency of model. Once mesh is defined, we assigned a material for every part of device [5]. Finally, the location of electrodes and doping level in each region is specified. After this we specify material parameters like permittivity, bandgap, carrier concentration, density of state in order to obtain electrical and optical characteristics of OLED. Furthermore, the polymer mobility parameters including carrier's mobility, thermal activation energy at zero electric field for the Poole-Frenkel-like mobility model and the workfunction for electrodes were also specified. Table 1 contains list of various parameters used for simulation purpose of P3HT based OLED.

Table 1: Parameters Used in P3HT- Based OLED Simulation

\begin{tabular}{|l|c|}
\hline \multicolumn{1}{|c|}{ Parameters } & Values \\
\hline \hline Thickness & $50 \mathrm{~nm}$ \\
\hline Electron affinity & $3.5 \mathrm{eV}$ \\
\hline Bandgap & $1.7 \mathrm{eV}$ \\
\hline Dielectric constant $(\varepsilon)$ & 3 \\
\hline Hole mobility $(\mu h)$ & $1 \times 10^{-2} \mathrm{~cm}^{2} / \mathrm{Vs}$ \\
\hline Electron mobility $(\mu n)$ & $1 \times 10^{-4} \mathrm{~cm}^{2} / \mathrm{Vs}$ \\
\hline $\begin{array}{l}\text { Effective density of states in the } \\
\text { conduction band (Nc) }\end{array}$ & $2 \times 10^{21} \mathrm{~cm}^{-3}$ \\
\hline $\begin{array}{l}\text { Effective density of states in the } \\
\text { valance band (Nv) }\end{array}$ & $2 \times 10^{21} \mathrm{~cm}^{-3}$ \\
\hline Accepter concentration & $5.75 \times 10^{17} \mathrm{~cm}^{-3}$ \\
\hline $\begin{array}{l}\text { Activation energy at zero electric } \\
\text { field for holes (deltaep.pfmob) }\end{array}$ & $1.792 \times 10^{-2} \mathrm{eV}$ \\
\hline $\begin{array}{l}\text { Hole Poole-Frenkel factor } \\
\text { (betap.pfmob) }\end{array}$ & $7.758 \times 10^{-5} \mathrm{eV}^{-\mathrm{cm} / \mathrm{V})^{1 / 2}}$ \\
\hline
\end{tabular}




\section{Physical Models}

For OLEDs, several organic models like PooleFrenkel-like mobility model, Langevin bimolecular recombination model and the singlet exciton model are used to describe the charge transport in the OLEDs. Poole-Frenkel-Like mobility model and Langevin recombination model are used to describe the transport and recombination mechanism of the polymer respectively, while the singlet exciton model is used to calculate the radiative rate for luminescence due to the Langevin recombination in the OLED.

The Poole-Frenkel mobility model describes the charge transport through the sub-bandgap density of state [6] and expressed by the following equations

$\mu_{n}(E)=\mu_{n 0} \exp \left(\frac{-D E L T A E N . P F M O B}{k T n e f f}+\left(\frac{B E T A N . P F M O B}{k T n e f f}-\right.\right.$ GAMMAN.PFMOB $\left.) \sqrt{|E|}\right)$

$\mu_{p}(E)=\mu_{p 0} \exp \left(\frac{-D E L T A E P . P F M O B}{k T n e f f}+\left(\frac{B E T A P . P F M O B}{k T n e f f}-G A M M A P . P F M O B\right) \sqrt{|E|}\right)$

where $\mu_{\mathrm{n}}(\mathrm{E})$ and $\mu_{\mathrm{p}}(\mathrm{E})$ are the Poole-Frenkel mobilities for electrons and holes respectively, $\mu_{n 0}$ and $\mu_{p 0}$ are the zero field mobilities for electrons and holes respectively, and $\mathrm{E}$ is the electric field. DELTAEN.PFMOB and DELTAEP.PFMOB are the activation energy at zero electric field for electrons and holes respectively. BETAN.PFMOB is the electron Poole-Frenkel factor, and BETAP.PFMOB is the hole Poole-Frenkel factor. Tneff and Tpeff are the effective temperature for electrons and holes respectively.

Due to the strong dependence on the electric field, the Poole-Frenkel mobility model can cause convergence problems. To increase the stability of the Poole-Frenkel mobility model, the following equations are used [5].

$$
\begin{aligned}
& \mu_{p}(E)=\frac{1}{\frac{1}{\mu_{p}(E)}+\frac{1}{\mu_{p \lim }(E)}} \\
& \mu_{n}(E)=\frac{1}{\frac{1}{\mu_{n}(E)}+\frac{1}{\mu_{n \lim }(E)}}
\end{aligned}
$$

where $\mu_{\mathrm{n}}(\mathrm{E})$ and $\mu_{\mathrm{p}}(\mathrm{E})$ are mobilities for electrons and holes respectively, $\mu_{\text {nlim }}(\mathrm{E})$ and $\mu_{\text {plim }}(\mathrm{E})$ are limiting mobility calculated from the thermal velocities.
The distribution of singlet excitons is used to infer the radiative rates for luminescense or phosphorescence in organic material LEDs. In Atlas, we can self-consistently solve the singlet exciton continuity equations along with the electron and hole drift diffusion equations. The singlet exciton continuity equation is given by [6]

$$
\begin{aligned}
& \frac{d S(x, y, t)}{d t}=\left[R S T . E X C I T O N R_{L n, p}+\right. \\
& (F . G E N S I N G L E T(x, y, z, t, n, p))+ \\
& \left(\frac{R S T \cdot T T \cdot E X C I T O N}{1+R S T \cdot T T \cdot E X C I T O N} \text { KTT.EXCITON } T^{2}(x, y, t)\right)- \\
& \text { (KISC. EXCITON S }(x, y, t))-(\text { KST.EXCITON } \\
& S(x, y, t) T(x, y, t)-\left(K_{d d} S(x, y, t)\right) \\
& \text {-KSP. EXCITON } S(x, y, t)(n(x, y, t)+p(x, y, t)- \\
& \left(\frac{K S S . E X C I T O N}{2} S^{2}(x, y, t)\right)-\text { KNRS.EXCITON } \\
& S(x, y, t)-\frac{\text { PHEFF.EXCITON }}{\text { TAUS.EXCITON }} S(x, y, t)+D_{S} \partial S(x, y, t)- \\
& Q(x, y) S(x, y, z)-R_{D n, p} S(x, y, t)+G_{P h} \\
& \text { QE.EXCITON] }
\end{aligned}
$$

where,

$S(x, y, t), \quad T(x, y, t)=$ Spatial and temporal singlet exciton and triplet exciton density respectively.

RST.EXCITON $=$ Fraction of singlets formed during Langevin recombination,

RST.TT.EXCITON = Fraction of singlets formed during triplet-triplet annihilation,

$R L n, p=$ Langevin recombination rate

$n(x, y, t)$ and $p(x, y, t)=$ Electron concentration and hole concentration,

TAUS.EXCITON TAUT.EXCITON = Singlet radiative decay lifetime and Triplet radiative decay lifetime respectively.

KISC.EXCITON $=$ Intersystem crossing constant

KST.EXCITON $=$ Singlet-triplet constant

KSP.EXCITON $=$ Singlet-polaron constant

KTT.EXCITON $=$ Triplet-triplet constant 
KNRS.EXCITON and KNRT.EXCITON = Singlet non-radiative decay constant and triplet non-radiative decay constant respectively.

$k d d=$ Dipole-dipole exciton transfer rate (Forster)

$Q(x, y)=$ Metal quenching rate

PHEFF.EXCITON $=$ Host photoluminescence quantum efficiency.

$R D n, p=$ Exciton dissociation rate

$G p h=$ Photoabsorption rate

QE.EXCITON $=$ Proportion of absorbed photons that is responsible for generating singlets

The bimolecular recombination rate is described by Langevin recombination rate that is given by the following equation

$$
R_{L n, p}=r_{L}(x, y, t)\left(n p-n i^{2}\right)
$$

where ni is intrinsic carrier concentration, $r_{L}(x, y, t)$ is Langevin recombination rate coefficient.

The Langevin recombination rate coefficient expressed as [7]

$$
R_{L}(x, y, t)=A \text { LANGEVIN } \frac{q\left[\mu_{n}(E)+\mu_{p}(E)\right]}{\varepsilon_{r} \varepsilon_{0}}
$$

where $\varepsilon_{r}$ is relative permittivity, $\varepsilon_{0}$ is absolute permittivity, A.Langevin specifies the prefactor for the bimolecular Langevin recombination model. By default, the value of model parameter A.Langevin is 1 .

OLED structure and Langevin recombination using Silvaco TCAD tonyplot is shown in Figure $\mathbf{4}$ and $\mathbf{5}$ respectively.

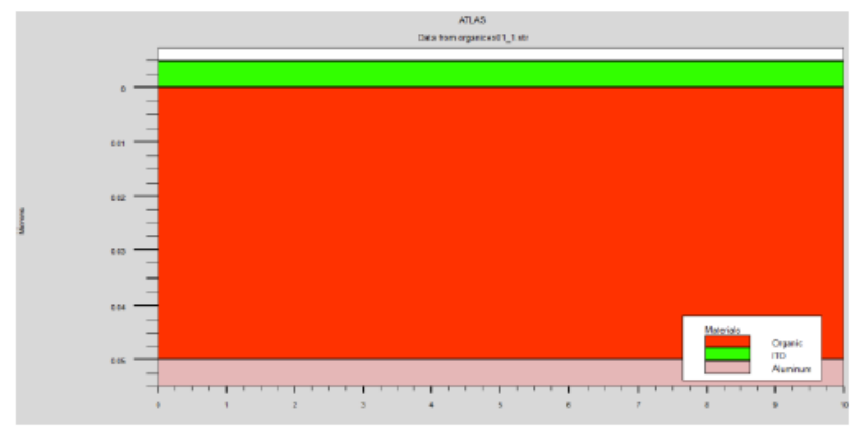

Figure 4: Structure of OLED in Silvaco Atlas.

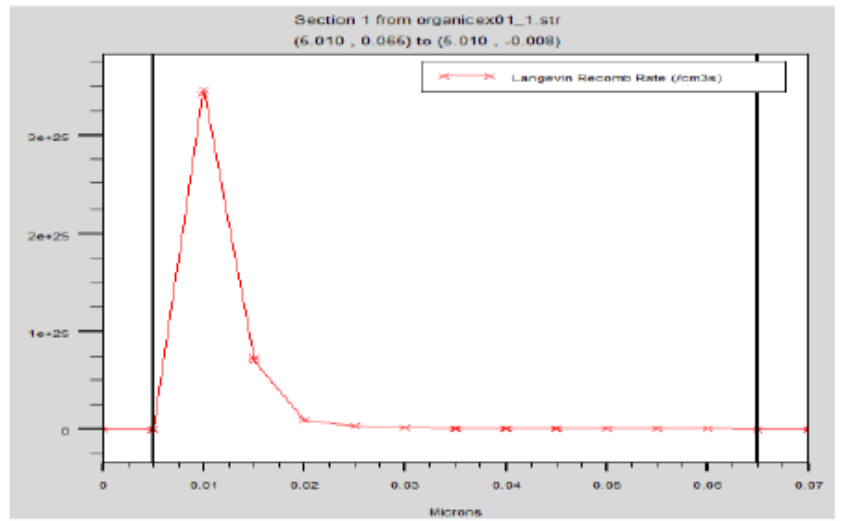

Figure 5: Langevin recombination rate.

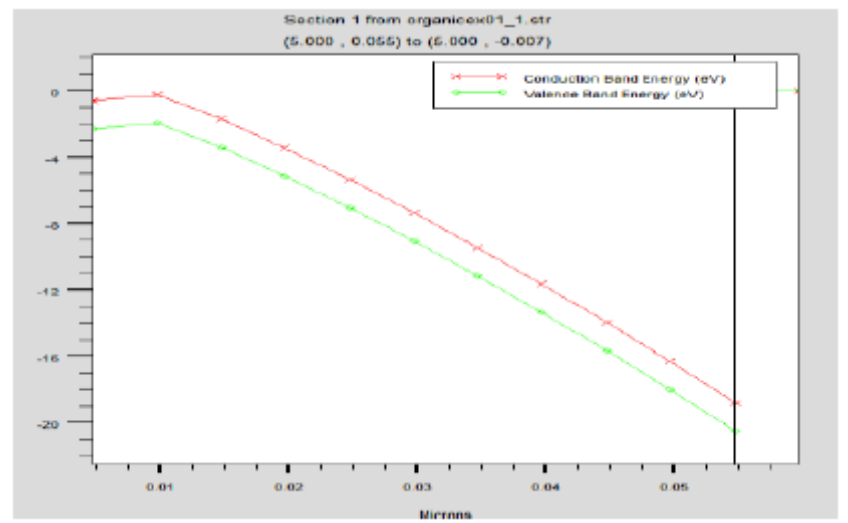

Figure 6: Conduction band energy and valence band energy.

Conduction band energy and valence band energy is given in Figure 6 . Figure 7 shows 1D-mode simulated singlet exciton density distribution.

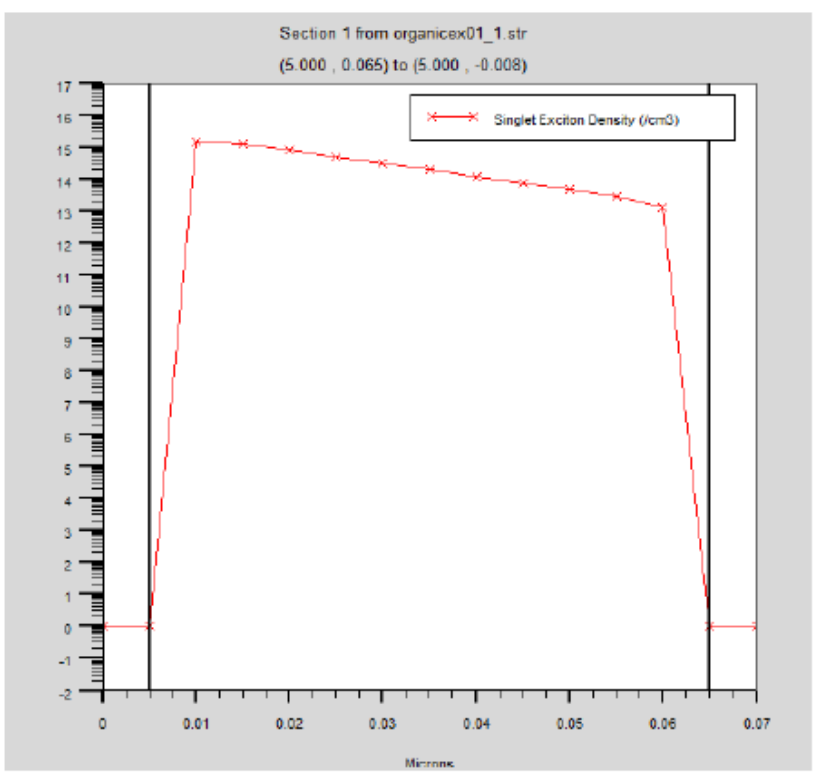

Figure 7: 1D-mode simulated singlet exciton density distribution. 


\section{RESULTS AND DISCUSSION}

I-V characteristic, luminescence power and current density versus electric field are obtained to estimate the performance of the OLED. The electrical characteristics of the device were obtained by changing the desired parameters (thickness and doping concentration) in the Silvaco TCAD Atlas file and the results of simulation are visualized in Tonyplot.

Figures $\mathbf{8}, \mathbf{9}$ and 10 represents I-V characteristics, luminescent power and electric field vs. current density of OLED for different thicknesses of emitting layer (P3HT) $(60,120,180$ and $240 \mathrm{~nm})$ respectively.

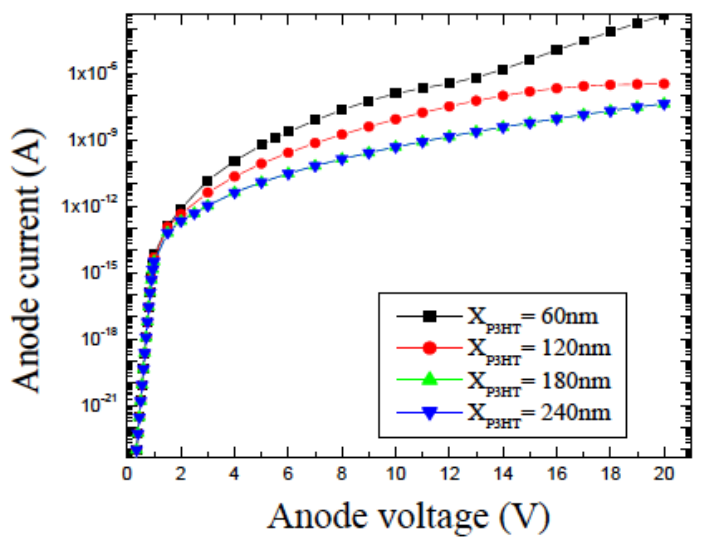

Figure 8: I-V characteristics of OLED for different thickness of emitting layer (P3HT)

It is evident from the figure that the device "turnson" at about 1.2 Volt and as the anode voltage increases, the potential luminescent power increases. It is also clear from the figure that the intensity of light decrease as the thickness of emitting layer (P3HT) increases thus it may concluded that it is better to make emitting layer thinner.

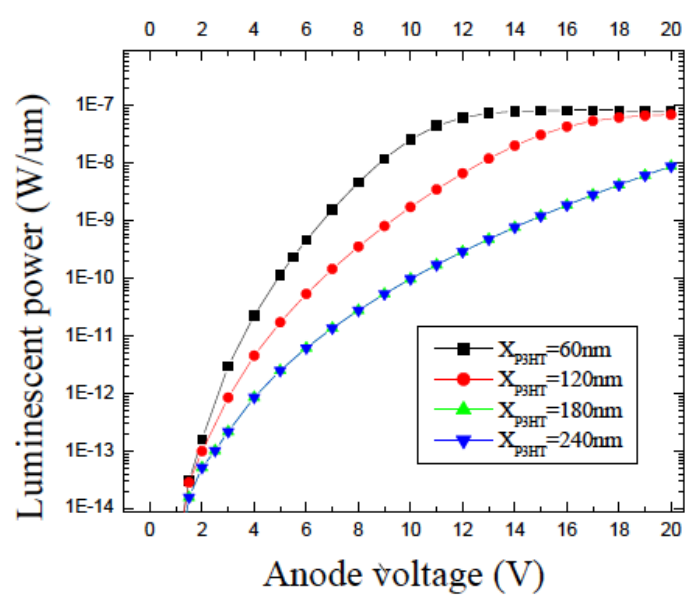

Figure 9: Luminescent power vs anode voltage for different thickness of $\mathrm{P} 3 \mathrm{HT}$

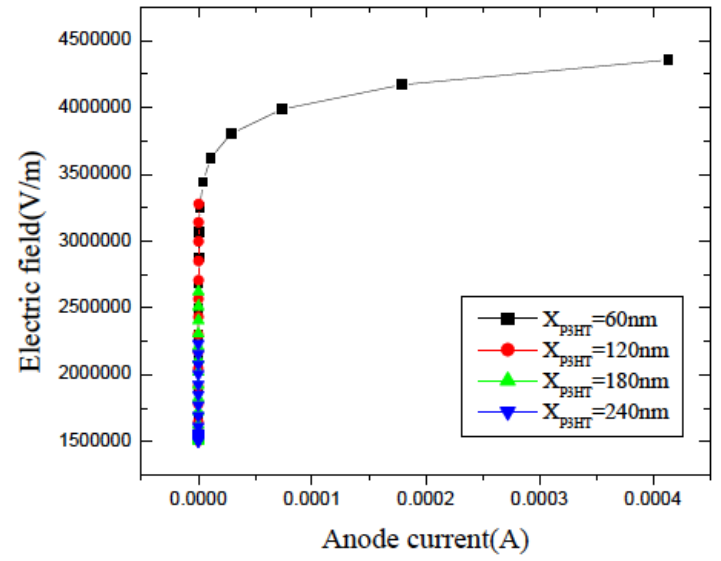

Figure 10: Electric field vs. current density for different thickness of P3HT

Similarly, Figures 11, 12 and 13 represents I-V characteristics, luminescent power and electric field vs. current density of OLED for different doping concentration of $\mathrm{P} 3 \mathrm{HT}$ respectively.

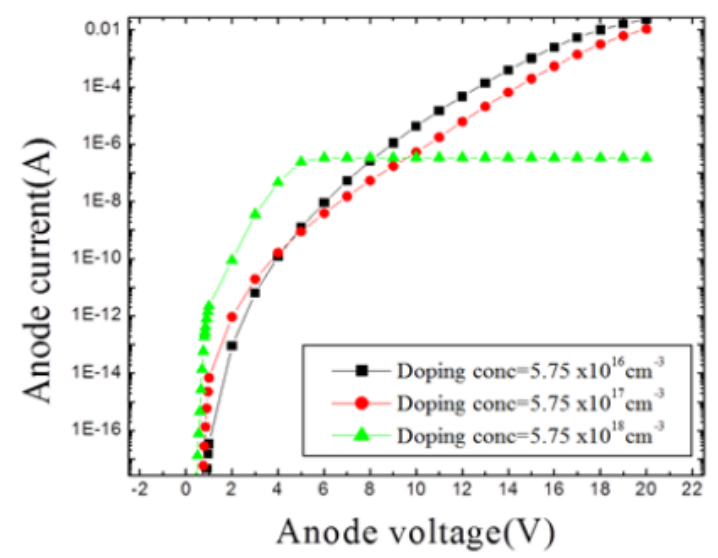

Figure 11: I-V Characteristics for different doping concentration.

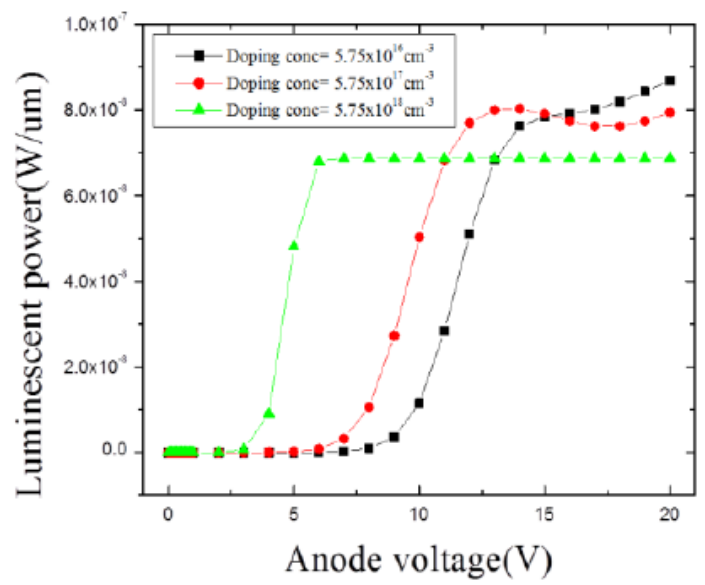

Figure 12: Luminescent power vs anode voltage for different doping concentration of P3HT. 


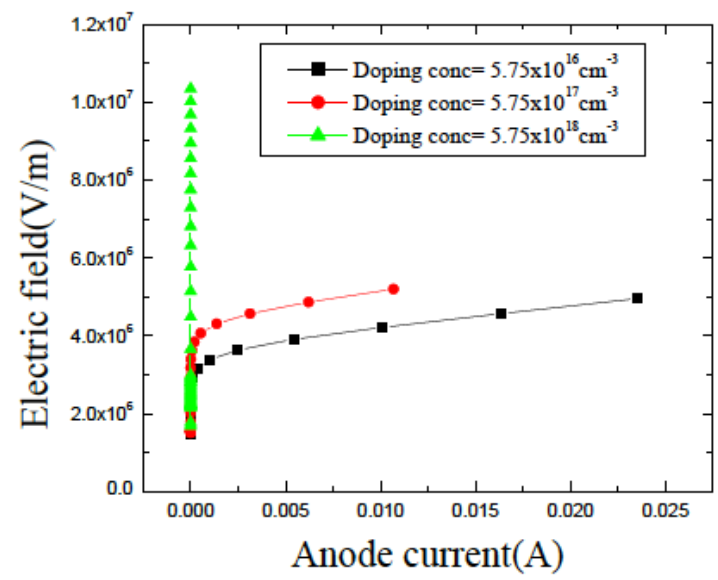

Figure 13: Electric field vs. anode current for different doping concentration.

\section{CONCLUSION}

In this paper, the structure, materials and operation principle of OLED with P3HT as the emitting layer is presented. 1D and 2D simulation of OLED were conducted using Silvaco TCAD software to investigate the device and predict its electrical and optical characteristics. The process of simulation is based on solving the differential equations describing the transport and recombination mechanism of the polymers and calculating the radiative rate for luminescence due to Langevin recombination in the OLED. Using TCAD tools we have obtained luminescence power characteristics, current density versus electric field, current density versus voltage and I-V characteristics for different thicknesses and doping concentration of emitting layer (P3HT). Simulations with varying $\mathrm{P} 3 \mathrm{HT}$ thickness showed that the decreasing thickness results in improvement of device characteristics.

The performed simulation allowed us to look and analyze physical processes inside the structure for better understanding the operation principles of OLED. Furthermore, the model helps us to optimize the diode design and to avoid time- consuming and costing experiments on real devices, thus streamlining the design process.

\section{REFERENCES}

[1] "OLEDs Replacing LCDs in Mobile Phones", Electronic News 2005.

[2] Silvaco TCAD, www.silvaco.com 2016.

[3] Mahmoud Al-Sa'di, Frank Jaiser, Sergey Bagnich, Thomas Unger, James Blakesley, et al., "Electrical and optical simulations of a polymer based phosphorescent organic light-emitting diode with high efficiency", Polymer Physics 2012.
Donghwa Shin, Naehyuck Chang, "Dynamic driver supply voltage scaling for organic light emitting diode displays", IEEE Trans. On CAD of IC and System 2012.

IA. Lysenko, LA. Patrashanu, DD. Zykov, "Organic Light Emitting Diode Simulation Using Silvaco TCAD Tools" International Siberian Conference on Control and Communications (SIBCON) 2016. https://doi.org/10.1109/SIBCON.2016.7491782

[6] Atlas User's Manual, www.silvaco.com

[7] Blom, PWM, MJM. de Jong, and S. Breedijk, "Temperature Dependent Electron-Hole Recombination in Polymer LightEmitting Diodes", Applied Physics Letters 1997; 71(7): 930932. https://doi.org/10.1063/1.119692

[8] Nidhi Sinha, Varun Chandra and ADD. Dwivedi, "TCAD Based Assessment and Performance Optimization of $\mathrm{ZnO} / \mathrm{Si}$ Heterojunction Based Thin-film Solar Cell" I International Journal of Advanced Applied Physics Research 2016; 3(2): 34-39.

[9] Nidhi Sinha, Varun Chandra and ADD Dwivedi" Modeling and Simulation of $\mathrm{n}-\mathrm{ZnO} / \mathrm{i}-\mathrm{ZnO} / \mathrm{Si}$ p-i-n Heterojunction Solar Cell " International Journal of Microelectronics and Digital integrated circuits 2016; 2(2): 1-5.

[10] Sumit Vyas, Shaivalini Singh and ADD. Dwivedi" Deposition and Characterization of $\mathrm{ZnO}$ Thin Films by Thermal Vacuum Thermal Evaporation Method for Photoconductive Sensor Application " International Journal of Microelectronics and Digital integrated circuits, 2016; 2: 1-7,

[11] ADD. Dwivedi, Anjan Chakravorty, R. D'Esposito, AK. Sahoo, S. Fregonese and T. Zimmer "Effect of BEOL on Self-heating and mutual thermal coupling in SiGe multi finger HBTs" Solid State Electronics 2016; 115: 1-6. https://doi.org/10.1016/i.sse.2015.09.016

[12] ADD. Dwivedi, R. D'Esposito, AK. Sahoo, S. Fregonese and T. Zimmer " A study on self-heating and mutual thermal coupling in SiGe multi-finger HBTs " Journal of Electronic Materials 2016; 45(11): 5612-5620. https://doi.org/10.1007/s11664-016-4728-6

[13] S. Patel, ADD. Dwivedi and P. Chakrabarti, "Numerica simulation of GaAs based MISFET photodetector for optical communication" International Journal of Microelectronics and Digital integrated circuits 2016; 2(1): 17-25.

[14] ADD. Dwivedi and P. Chakrabarti, "Analytical Modeling and Numerical Simulation of $\mathrm{Hg}_{1-x} \mathrm{Cd}_{x} T \mathrm{e}$ based $\mathrm{N}^{+} \mathrm{n}^{0} \mathrm{p}^{+}$ Photodetector for MWIR Free Space Optical Communication," International Journal of Advanced Applied Physics Research 2015; 2: 20-27. https://doi.org/10.15379/2408-977X.2015.02.02.4

[15] ADD. Dwivedi, Rajeev Dhar Dwivedi, Raghvendra Dhar Dwivedi, Sumit Vyas and P. Chakrabarti, "Numerical simulation of P3HT based Organic Thin Film Transistors (OTFTs)" International Journal of Microelectronics and Digital integrated circuits 2015; 1(2): 13-20.

[16] ADD. Dwivedi, A. Pranav, Gaurav Gupta and P. Chakrabarti, "Numerical Simulation of $\mathrm{HgCdTe}$ based simultaneous MWIR/LWIR photodetector for free space optical communication" International Journal of Advanced Applied Physics Research 2015; 2: 37-45. https://doi.org/10.15379/2408-977X.2015.02.01.5

ADD. Dwivedi, "Numerical Simulation and Spice Modeling of Organic Thin Film Transistors (OTFTs)" International Journal of Advanced Applied Physics Research 2014; 1: 14-21. https://doi.org/10.15379/2408-977X.2014.01.02.3

[18] ADD. Dwivedi, "Analytical Modeling and Numerical Simulation of $\mathrm{P}^{+}-\mathrm{Hg}_{0.69} \quad \mathrm{Cd}_{0.31} \mathrm{Te} / \mathrm{n}-\mathrm{Hg}_{0.78} \mathrm{Cd}_{0.22} \mathrm{Te} / \mathrm{CdZnTe}$ Heterojunction Photodetector for LWIR Free Space Optical Communication System," Journal of Applied Physics 2011; 110: 043101

https://doi.org/10.1063/1.3615967 
[19] ADD. Dwivedi, "Analytical Modeling and ATLAS Simulation of $\mathrm{p}^{+}-\mathrm{Hg}_{0.78} \quad \mathrm{Cd}_{0.22} \mathrm{Te} / \mathrm{n}-\mathrm{Hg}_{0.78} \mathrm{Cd}_{0.22} \mathrm{Te} / \mathrm{CdZnTe}$ Homojunction Photodetector for LWIR Free Space Optical Communication System," Journal of Electron Devices 2011; 9: 396-404.

[20] ADD. Dwivedi and P. Chakrabarti, "Sensitivity Analysis of an $\mathrm{Hg}_{1-x} \mathrm{Cd}_{\mathrm{x}} \mathrm{Te}$ based Photoconductive Receiver for Long Wavelength Free space optical communication at $9.6 \mu \mathrm{m}$," Journal of Electron Devices 2011; 9: 390-395.

[21] ADD. Dwivedi, A. Mittal, A. Agrawal and P. Chakrabarti, "Analytical Modeling and ATLAS Simulation of $\mathrm{N}^{+}-\ln P / \mathrm{n}^{0}$ $\mathrm{In}_{0.53} \mathrm{Ga}_{0.47} \mathrm{As} / \mathrm{p}^{+} \mathrm{In}_{0.53} \mathrm{Ga}_{0.47}$ As p-i-n Photodetector for Optical Fiber Communication," Infrared Physics \& Technology 2010; 53(4): 236-245. https://doi.org/10.1016/j.infrared.2010.03.003

[22] ADD. Dwivedi and P. Chakrabarti, "Analytical Modeling and ATLAS Simulation of $\mathrm{N}^{+}-\mathrm{Hg}_{0.69} \quad \mathrm{Cd}_{0.31} \mathrm{Te} \quad / \mathrm{n}^{0}-\mathrm{Hg}_{0.78}$ $\mathrm{Cd}_{0.22} \mathrm{Te} / \mathrm{p}^{+} \mathrm{Hg}_{0.78} \quad \mathrm{Cd}_{0.22} \mathrm{Te}$ p-i-n Photodetector for Long wavelength Free Space Optical Communication," Optoelectronics and Advanced Materials-Rapid Communications (OAM-RC) 2010; 4(4): 480-497.

[23] Ghusoon M. Ali, ADD. Dwivedi, S. Singh, and P. Chakrabarti "Interface properties and junction behavior of Pd contact on ZnO thin film grown by vaccum deposition technique," Physica Status Solidi (PSS) C 2010; 7: 252-255.

[24] Arun Kumar Singh, ADD. Dwivedi, P. Chakrabarti and Rajiv Prakash, "Electronic and Optical Properties of Electrochemically Polymerized Polycarbazole/Aluminum Schottky Diodes," Journal of Applied Physics 2009; 105: 114506. https://doi.org/10.1063/1.3139277

[25] ADD. Dwivedi and P. Chakrabarti, "Sensitivity analysis of an $\mathrm{HgCdTe}$ based photovoltaic receiver for long- wavelength free space optical communication systems" Optoelectronics Letters 2009; 5: 21-25.

https://doi.org/10.1007/s11801-009-8123-x

[26] AK. Singh, Rajiv Prakash, ADD. Dwivedi and P. Chakrabarti, "Electronic Properties and Junction Behaviour of Polyanthranilic Acid / Metal Contacts." IEEE Electron Device Letter 2008; 29(6): 571-574.

https://doi.org/10.1109/LED.2008.922730

[27] AK. Singh, Rajiv Prakash, ADD. Dwivedi and P. Chakrabarti, "Electronic Properties and Junction Behaviour of Micro and Nanometer Sized Polyanthranilic Acid / Metal Contacts." Synthetic Metals 2008; 158: 939-945. https://doi.org/10.1016/j.synthmet.2008.06.020

[28] ADD. Dwivedi and P. Chakrabarti, "Modeling and Analysis of Photoconductive Detectors based on $\mathrm{Hg}_{1-x} \mathrm{Cd}_{\mathrm{x}} \mathrm{Te}$ for free Space Optical Communication." Optical and Quantum Electronics 2007; 39: 627-641. https://doi.org/10.1007/s11082-007-9122-4

[29] JD. Aguirre-Morales, S. Fregonese, ADD. Dwivedi, T. Zimmer, MS. Khenissa and H. Happy "Towards Amplifier Design with a SiC Graphene Field-Effect Transistor" in 2015 Joint International EUROSOI Workshop and International Conference on Ultimate Integration on Silicon (EUROSOIULIS-2015) 2015; 26-28, - held at Bologna, Italy.

[30] ADD. Dwivedi, Arun Kumar Singh, Rajiv Prakash and $P$ Chakrabarti, "A Comparative Study of Polyanthranilic Acid (PANA)/Metal and Polycarbarzale (PCz)/Metal Contacts for Electronic and Optoelectronic Applications," $2^{\text {nd }}$ National Workshop on Advance Optoelectronic Materials and Devices, AOMD-2008, held at centre for research in microelectronics, Department of Electronics Engineering, I T BHU, Varanasi-221005, pp. 56-64, during 22-24, 2008.

\section{http://dx.doi.org/10.15379/2408-977X.2018.05.01.02}

(c) 2018 Raj and Dwived; Licensee Cosmos Scholars Publishing House.

This is an open access article licensed under the terms of the Creative Commons Attribution Non-Commercial License

(http://creativecommons.org/licenses/by-nc/3.0/), which permits unrestricted, non-commercial use, distribution and reproduction in any medium, provided the work is properly cited. 